\title{
Erratum to: Pulses and snakes in Ginzburg-Landau equation
}

\author{
Stefan C. Mancas • Roy S. Choudhury
}

Published online: 9 October 2014

(C) Springer Science+Business Media Dordrecht 2014

\section{Erratum to: Nonlinear Dyn DOI 10.1007/s11071-014-1686-5}

The following information was omitted in the original publication: The proof at the beginning of Section 3 applies only to regular Hopf bifurcations involving a pair of bifurcating (pure imaginary) eigenvalues.
Note however that, although simple Hopf bifurcations are impossible, the well-known HamiltonianHopf bifurcation involving four bifurcating eigenvalues is of course ubiquitous in Hamiltonian systems. Note that this Hamiltonian-Hopf bifurcation cannot occur in our three-dimensional system though.

The online version of the original article can be found under doi:10.1007/s11071-014-1686-5.

S. C. Mancas $(\varangle)$

Department of Mathematics, Embry-Riddle Aeronautical

University, Daytona Beach, FL 32114, USA

e-mail: mancass@erau.edu

R. S. Choudhury

Department of Mathematics, University of Central Florida,

Orlando, FL 32816, USA

e-mail: choudhur@longwood.cs.ucf.edu 